\title{
Ultrafast dephasing of coherent optical phonons in atomically controlled $\mathrm{GeTe} / \mathrm{Sb}_{2} \mathrm{Te}_{3}$ superlattices
}

\author{
Muneaki Hase, ${ }^{1,2, *}$ Yoshinobu Miyamoto, ${ }^{1}$ and Junji Tominaga ${ }^{3}$ \\ ${ }^{1}$ Institute of Applied Physics, University of Tsukuba, 1-1-1 Tennodai, Tsukuba 305-8573, Japan \\ ${ }^{2}$ PRESTO, Japan Science and Technology Agency, 4-1-8 Honcho, Kawaguchi, Saitama 332-0012, Japan \\ ${ }^{3}$ Center for Applied Near-field Optics Research, National Institute of Advanced Industrial Science and Technology, \\ Tsukuba Central 4, 1-1-1 Higashi, Tsukuba 305-8562, Japan
}

(Received 3 February 2009; revised manuscript received 20 April 2009; published 21 May 2009)

\begin{abstract}
Femtosecond dynamics of coherent optical phonons in $\mathrm{GeTe} / \mathrm{Sb}_{2} \mathrm{Te}_{3}$ superlattices (SLs), a new class of semiconductor SLs with three different states, have been investigated by using a reflection-type pump-probe technique at various lattice temperatures. The time-resolved transient reflectivity obtained in as-grown SLs exhibits the coherent $A_{1}$ optical modes at 5.10 and $3.78 \mathrm{THz}$ while only the single $\mathrm{A}_{1}$ mode at $3.68 \mathrm{THz}$ is observed in annealed SLs. The decay rate of the $\mathrm{A}_{1}$ mode in annealed SLs is strongly temperature dependent while that in as-grown SLs is not temperature dependent. This result indicates that the damping of the coherent $A_{1}$ phonons in amorphous SLs is governed by the phonon-defect (vacancy) scattering rather than the anharmonic phonon-phonon coupling.
\end{abstract}

DOI: 10.1103/PhysRevB.79.174112

PACS number(s): 78.47.J-, 63.20.kp, 63.50.-x, 68.35.Rh

One of the most common materials for optical recording media is $\mathrm{Ge}_{2} \mathrm{Sb}_{2} \mathrm{Te}_{5}$ (GST) in which phase transition between crystalline and amorphous phases serve rewritable recording. ${ }^{1,2}$ Recently, extensive theoretical investigation on the mechanism of the phase change in GST have been made using molecular-dynamics simulations. ${ }^{3-5}$ In addition, experimental studies using extended $\mathrm{x}$-ray absorption fine structure (XAFS) and Raman-scattering measurements have examined dynamics of phase transition in GST, ${ }^{6-9}$ suggesting that the structure of amorphous GST can be described as a cross section of a distorted rocksalt structure with vacancies and the amorphization of GST is due to an umbrella flip of Ge atoms from an octahedral position into a tetrahedral one. Moreover, Sun et al. ${ }^{4}$ theoretically proposed that the vacancies in the crystalline (cubic) GST are highly ordered and layered followed by the recent prediction of the formation of large voids in the amorphous GST films. ${ }^{10}$ The experimental information on the existence of vacancies from the lattice dynamical point of view, however, has not been explored.

One of the advantages of GST as the optical recording media is its high-speed switching of read-write characteristics whose time scale has been believed to be less than a nanosecond. In order to understand and control the rapid phase change in GST, a time-resolved study of phonon dynamics in GST is strongly demanded, however, the timeresolved studies are still very few. ${ }^{11}$ Moreover, a new class of semiconductor superlattices $\left(\mathrm{GeTe} / \mathrm{Sb}_{2} \mathrm{Te}_{3}\right)$ with three different states has recently been proposed which will enable us to realize reversible transition among the three states by the means of the irradiation of laser pulses. ${ }^{12}$

The coherent phonon spectroscopy (CPS) is a powerful tool to study ultrafast dynamics of structural phase transitions, occurring within picosecond and femtosecond time scales and in fact it has been applied to semimetals, ${ }^{13}$ ferroelectric materials, ${ }^{14,15}$ and Mott insulators. ${ }^{16,17}$ In the CPS, the pump pulse impulsively generates coherent lattice vibration through real or virtual electronic transitions. The pulse length $(\Delta)$ used should be much shorter than the time period of the lattice vibration, so it is typically $\Delta \leq 100$ fs to excite phonons with terahertz $(\mathrm{THz})$ frequency. It has been shown that the dephasing of the coherent optical phonon is very sensitive to the density of vacancy. ${ }^{18}$ Regarding to $\mathrm{Ge}_{2} \mathrm{Sb}_{2} \mathrm{Te}_{5}$, Först et al. ${ }^{11}$ investigated dynamics of phase transition in GST films by using the CPS and found that the appearance of the phonon modes was significantly modified upon the structural change among amorphous, cubic, and hexagonal structures. In their study, the observed phonon modes in GST films were always strongly damped modes with a dephasing time of less than several picoseconds, however, the dynamics of the dephasing of coherent phonons in GST compounds have not yet been revealed.

In this paper, we present ultrafast dephasing dynamics of coherent optical phonons observed in atomically controlled $\mathrm{GeTe} / \mathrm{Sb}_{2} \mathrm{Te}_{3}$ superlattices (SLs) at various lattice temperatures. Our motivation for the use of the atomically controlled $\mathrm{GeTe} / \mathrm{Sb}_{2} \mathrm{Te}_{3} \mathrm{SLs}$ is based on the new structural model that $\mathrm{Ge}_{2} \mathrm{Sb}_{2} \mathrm{Te}_{5}$ is considered as superlattice, which consists of two units: one is a $\mathrm{Ge}_{2} \mathrm{Te}_{2}$ layer and the other is a $\mathrm{Sb}_{2} \mathrm{Te}_{3}$ layer. ${ }^{19,20}$ That indicates understanding the role of the flipflop transition of the Ge atom in the distorted simple-cubic unit cell will be a promising strategy toward the reversible transition by means of the irradiation of ultrashort laser pulses. Our experiments show that the frequency of the coherent $A_{1}$ optical modes decreases with increasing the lattice temperature. The decay rate (the inverse of the dephasing time) of the coherent $A_{1}$ mode increases as the lattice temperature increases in annealed (crystalline) SLs while that in as-grown (amorphous) SLs is almost constant over the wide temperature range. Our data demonstrate that randomly distributed vacancies or voids indeed exist in the amorphous phase while they become "ordered" in the crystalline phase. The differences of phonon dynamics in the GST film and $\mathrm{GeTe} / \mathrm{Sb}_{2} \mathrm{Te}_{3}$ SLs are also discussed.

A reflection-type pump-probe measurement using a modelocked Ti:sapphire laser $(\Delta=20$ fs and a central wavelength $850 \mathrm{~nm}$ ) was employed at the temperature range of 5-300 K. 


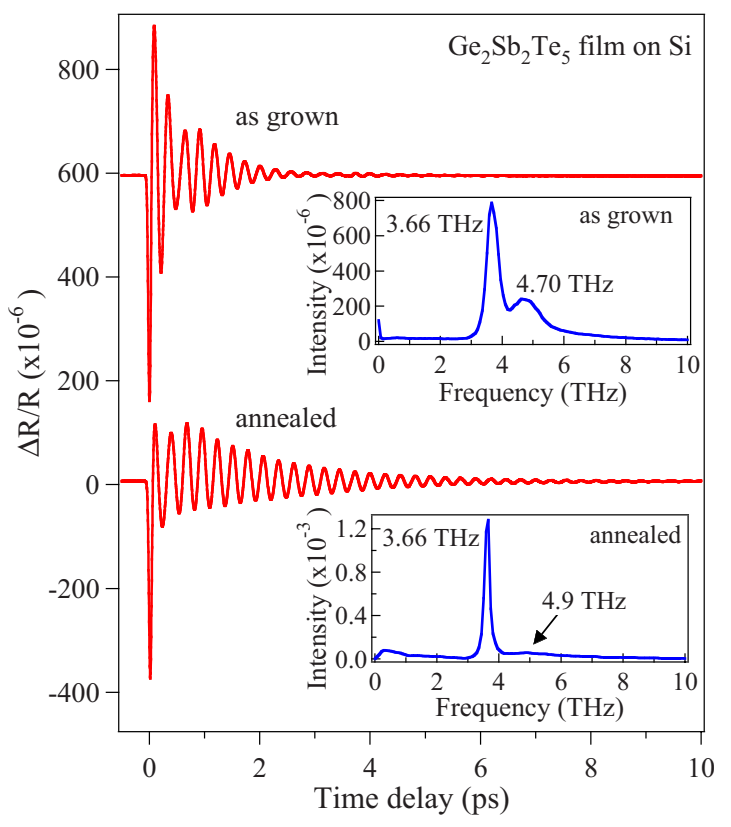

FIG. 1. (Color online) The TR signal observed in amorphous and crystalline $\mathrm{Ge}_{2} \mathrm{Sb}_{2} \mathrm{Te}_{5}$ films at $295 \mathrm{~K}$. The insets represent FT spectra obtained from the time-domain data.

This system enabled us to detect optical response up to 30 $\mathrm{THz}$ bandwidth. The average power of the pump and probe beams were fixed at 120 and $3 \mathrm{~mW}$, respectively, from which we estimated the pump fluence to be $284 \mu \mathrm{J} / \mathrm{cm}^{2}$ at 120 $\mathrm{mW}$. The optical-penetration depth of the laser light was estimated to be $\leq 50 \mathrm{~nm}$ at $1.46 \mathrm{eV}$ which is longer than the thickness of GST film. This fact indicates that we probe the whole depth of the films including the interface to Si substrate, however, the contribution from the Si substrate to the signal should be negligibly small because of extremely small optical-absorption strength in $\mathrm{Si}$ at $1.46 \mathrm{eV}$ photon energy. ${ }^{21}$ The samples used were GST film (18 nm thick) and thin film consisting of superlattice of $\mathrm{GeTe}$ and $\mathrm{Sb}_{2} \mathrm{Te}_{3}$ layers $\left(\mathrm{GeTe} / \mathrm{Sb}_{2} \mathrm{Te}_{3}\right)$ fabricated using a helicon-wave rf magnetron-sputtering machine on $\mathrm{Si}(100)$ substrate. The annealing of the as-grown $\mathrm{GeTe} / \mathrm{Sb}_{2} \mathrm{Te}_{3} \mathrm{SL}$ films at $503 \mathrm{~K}$ $\left(230{ }^{\circ} \mathrm{C}\right)$ for $10 \mathrm{~min}$ changed the amorphous states into the crystalline state. ${ }^{19}$ The TEM measurements confirmed that the $\mathrm{GeTe} / \mathrm{Sb}_{2} \mathrm{Te}_{3} \mathrm{SLs}$ have layered structures with clear interfaces. In addition, the structural change of the $\mathrm{GeTe} / \mathrm{Sb}_{2} \mathrm{Te}_{3} \mathrm{SLs}$ was evident from the increase in the reflectivity $(\sim 10 \%)$ after the annealing. The excitation of the GST and $\mathrm{GeTe} / \mathrm{Sb}_{2} \mathrm{Te}_{3}$ SLs with the $850 \mathrm{~nm}(=1.46 \mathrm{eV})$ laser pulse generates photo carriers across the narrow band gap of $\approx 0.5-0.7 \mathrm{eV} .{ }^{22}$ Transient reflectivity (TR) signal $(\Delta R / R)$ was measured as a function of the time delay $\tau$ after excitation pulse.

Figure 1 shows the time-resolved TR signal $(\Delta R / R)$ observed in $\mathrm{Ge}_{2} \mathrm{Sb}_{2} \mathrm{Te}_{5}$ films with amorphous (as-grown) and crystalline (annealed) phases at $295 \mathrm{~K}$. After the transient electronic response due to the excitation of photo carriers at $\tau=0$, coherent phonon oscillations with a few picoseconds dephasing time appear. Fourier-transformed (FT) spectra are obtained from the full scan of the time-domain data without
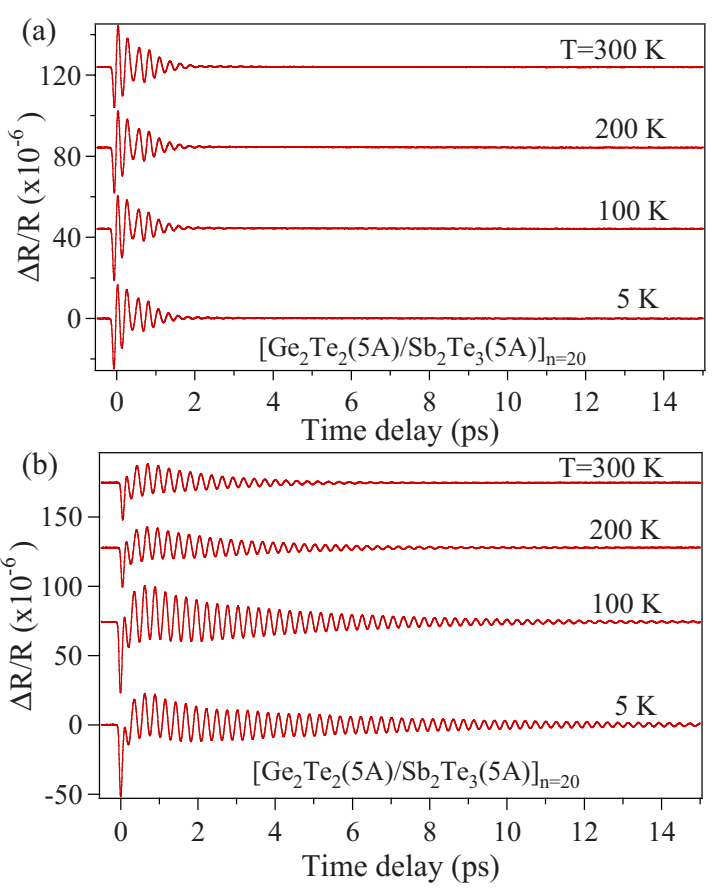

FIG. 2. (Color online) The TR signal observed (a) in amorphous and in (b) crystalline $\mathrm{GeTe} / \mathrm{Sb}_{2} \mathrm{Te}_{3}$ SLs at various temperatures.

any modification as shown in the inset of Fig. 1, in which the two broad peaks are observed at 4.70 and $3.66 \mathrm{THz}$ in amorphous film while the sharp peak at $3.66 \mathrm{THz}$ and a broad weaker band at $\approx 4.9 \mathrm{THz}$ are observed in crystalline film. These peaks in the amorphous film can be considered to be the $\mathrm{A}_{1}$ optical mode due to tetrahedral $\mathrm{GeTe}_{4}$ structure for the $3.66 \mathrm{THz}$ peak ${ }^{11}$ and the $\mathrm{A}_{1}$ optical modes due to disordered Te-Te chains ${ }^{7,11}$ or the $\mathrm{A}_{1}$ optical mode due to $\mathrm{Sb}_{2} \mathrm{Te}_{3}$ sublattice, as has recently been proposed, ${ }^{9}$ for the $4.70 \mathrm{THz}$ peak. The peaks in the crystalline phase at 3.66 and $\approx 4.9 \mathrm{THz}$ are almost in agreement with those observed by Först et al. ${ }^{11}$ although the peak at $2.0 \mathrm{THz}$ is not detected in the present study as observed in the past experiments. The difference in the FT spectra found in the crystalline films would be due to the condition of the sample; we annealed the amorphous GST film at $220^{\circ} \mathrm{C}$ while the coherent phonon was detected at elevated temperature of $160{ }^{\circ} \mathrm{C}$ in the past work, ${ }^{11}$ so the local structure of GST could be slightly different in each case.

Figure 2 compares the time-resolved TR signal observed in $\left[\mathrm{Ge}_{2} \mathrm{Te}_{2}(5 \AA) / \mathrm{Sb}_{2} \mathrm{Te}_{3}(5 \AA)\right]_{n=20}$ SL films with (a) amorphous and (b) crystalline phases at various temperatures; the total composition corresponds to $\mathrm{Ge}_{2} \mathrm{Sb}_{2} \mathrm{Te}_{5} \cdot{ }^{19}$ In Fig. 2(a) the coherent phonon oscillations with fast dephasing time of less than $\approx 1.5 \mathrm{ps}$ are observed and the dephasing time of the coherent phonon does not seem to depend on the lattice temperature. On the other hand, in Fig. 2(b) the coherent oscillation shows longer dephasing time than that in Fig. 2(a) and it is strongly temperature dependent.

The difference in these two samples is clearer in the corresponding FT spectra in which the two broad peaks are visible at 5.10 and $3.78 \mathrm{THz}$ in amorphous film in Fig. 3(a) while the dominant $3.68 \mathrm{THz}$ peak is observed in crystalline film in Fig. 3(b) both at 300 K. Here, FT spectra are obtained 

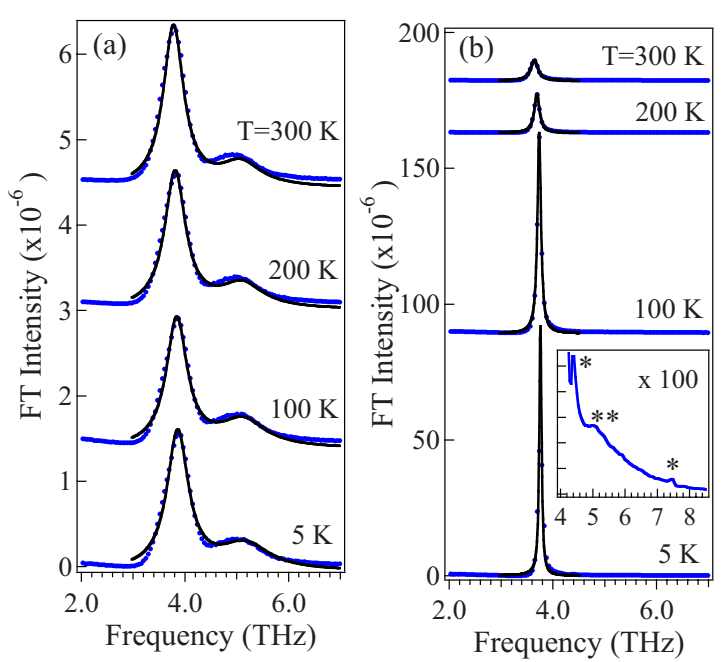

FIG. 3. (Color online) FT spectra obtained from the timedomain data in Fig. 2; (a) amorphous and (b) crystalline $\mathrm{GeTe} / \mathrm{Sb}_{2} \mathrm{Te}_{3} \mathrm{SLs}$ at various temperatures. The solid lines are the fit to the data with Lorentz functions. The inset in (b) represents magnified FT spectrum at $5 \mathrm{~K}$ in which two sharp peaks (*) and a broad peak $(* *)$ are detected.

from the full scan of the time-domain data in Fig. 2 without any modification. In our experiment, since the observed optical phonons in the amorphous films are localized modes, inhomogeneous damping would partly contribute to the ultrafast dynamics of the coherent optical phonons, as reported in glass materials. ${ }^{23}$ The inhomogeneous damping would include fluctuation of the environment of the local $\mathrm{A}_{1}$ modes resulting in the asymmetric line broadening of the FT spectra. The contribution from the inhomogeneous damping, however, would be negligibly small in the case of GST because the line shapes of the $\mathrm{A}_{1}$ modes are almost symmetric, which is similar to ion-irradiated $\mathrm{Bi}$ where phonon-vacancy scattering dominate the change in the dephasing time. The peaks in the amorphous phase can be assigned to the $A_{1}$ optical mode due to tetrahedral $\mathrm{GeTe}_{4}$ structure for the 3.78 THz peak and the $\mathrm{A}_{1}$ optical modes due to amorphous Te-Te chains or the $\mathrm{A}_{1}$ optical mode due to $\mathrm{Sb}_{2} \mathrm{Te}_{3}$ sublattice for the $5.10 \mathrm{THz}$ peak, as discussed in GST film in Fig. 1.9,11 Note that the peak at $3.68 \mathrm{THz}$ in crystalline phase is slightly lower than that observed in amorphous phase $(3.78 \mathrm{THz})$. The peak shift from 3.78 (amorphous) to $3.68 \mathrm{THz}$ (crystalline) could possibly be due to the change in the local structure of $\mathrm{GeTe}_{4}$ into $\mathrm{GeTe}_{6}{ }^{6}$ It is to be noted that in the crystalline SL a broad peak at $\approx 5.0 \mathrm{THz}$ and two sharp peaks at 4.37 and $\approx 7.5 \mathrm{THz}$ are detected, as shown in the inset of Fig. 3(b). The peak at $5.0 \mathrm{THz}$ would be due to the residual of the Te-Te chains or of the optical mode from $\mathrm{Sb}_{2} \mathrm{Te}_{3}$ sublattice. The peak at $4.37 \mathrm{THz}$ is not unknown at present but may be a confined $\mathrm{A}_{1}$ mode from the $\mathrm{Sb}_{2} \mathrm{Te}_{3}$ layer. The peak at $7.5 \mathrm{THz}$ is possibly due to $\mathrm{Ge}-\mathrm{Ge}$ stretching vibrations as observed in the Raman study. ${ }^{9}$

The coherent optical phonons in $\mathrm{GeTe} / \mathrm{Sb}_{2} \mathrm{Te}_{3}$ SLs with an amorphous phase exhibit significant shift of their frequency relative to the GST film at $\approx 300 \mathrm{~K}$. The frequency of the $\mathrm{A}_{1}$ optical mode at $3.78 \mathrm{THz}$ observed in amorphous
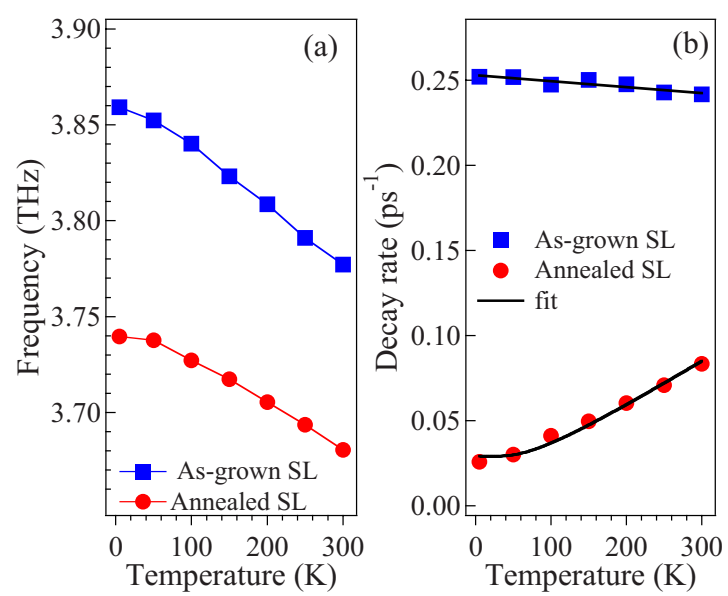

FIG. 4. (Color online) (a) the frequency and (b) the decay rate of the coherent $\mathrm{A}_{1}$ mode, which is localized in GeTe layer, in amorphous and crystalline $\mathrm{GeTe} / \mathrm{Sb}_{2} \mathrm{Te}_{3}$ SLs as the function of the lattice temperatures. In (b) the solid lines are the fit to the data with a linear function for the as-grown SL and the anharmonic decay model for the annealed SL.

SLs was originally at $3.66 \mathrm{THz}$ and that at $5.10 \mathrm{THz}$ was $4.70 \mathrm{THz}$ in the amorphous GST film. These phonon frequencies observed in SLs in amorphous phase are 3-7\% higher than those observed in the GST films and cannot be attributed to the confinement of the optical-phonon modes in each layers since the confinement of the optical phonons usually lower the frequency. ${ }^{24} \mathrm{~A}$ plausible explanation for the frequency shift is the volume expansion of the amorphous GST film, which is due to the randomly distributed vacancies in the amorphous phase, resulting in the reduction of the phonon frequency due to the longer bond lengths (smaller bond strength). ${ }^{21}$ In fact, based on the first-principle calculations, the volume expansion of $\approx 4 \%$ is expected due to the randomly distributed vacancies (or voids) in the amorphous phase of GST (Ref. 20); in amorphous SLs the structure is layered (ordered) and therefore the randomness of vacancies will be slightly compensated. In the crystalline phase, on the other hand, the single peak observed at $3.68 \mathrm{THz}$ in crystalline SLs is almost in the same position to that obtained in cubic GST films (3.66 THz in Fig. 1). This would demonstrate that the intrinsic structure of the cubic GST film is already the layered structure of $\left[-\left(\mathrm{Sb}_{2} \mathrm{Te}_{3}\right) \cdots(\mathrm{Ge}-\mathrm{Te}-\mathrm{Te}-\mathrm{Ge}) \cdots\right]_{n} \cdot{ }^{19}$

Figure 4 shows the frequency and the decay rate of the coherent $\mathrm{A}_{1}$ mode $\left(\mathrm{GeTe}_{4}\right.$ or $\mathrm{GeTe}_{6}$ modes) as a function of the lattice temperature. The decay rate of the crystalline phase increases with increasing the temperature while that in the amorphous phase is almost constant when the temperature is varied. The behavior of the decay rate in the crystalline phase is well explained by the anharmonic decay mode ${ }^{25}$ in which the optical phonon decays into the two acoustic phonons under the conservation of energy and momentum (see Fig. 5); the acoustic phonons with half the frequency of the optical mode $\left(\hbar \Omega_{0} / 2\right)$ and with opposite wave vectors ${ }^{25,26}$ 


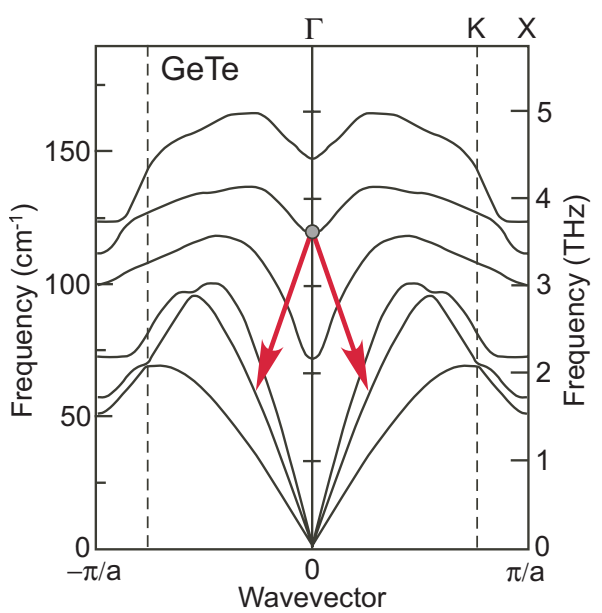

FIG. 5. (Color online) Phonon-dispersion relations of GeTe. Since the optical phonon discussed here is localized in GeTe layer we assume that the phonon dispersion of the $\mathrm{GeTe}_{4}$ or $\mathrm{GeTe}_{6}$ modes is similar to that of GeTe. The optical mode at $\approx 3.7 \mathrm{THz}$ can then relax into the two underlying acoustic phonons as shown by the arrows. From Ref. 31.

$$
\Gamma=\Gamma_{0}\left[1+\frac{2}{\exp \left(\frac{\hbar \Omega_{0} / 2}{k_{B} T}\right)-1}\right]
$$

Here $\Gamma_{0}$ is the effective anharmonicity as the fitting parameter and $k_{B}$ the Boltzmann constant. $\Gamma_{0}$ is determined to be $0.03 \mathrm{ps}^{-1}$. The good agreement of the time-domain data with the anharmonic decay model indicates that the damping of the coherent $\mathrm{A}_{1}$ mode in crystalline $\mathrm{GeTe} / \mathrm{Sb}_{2} \mathrm{Te}_{3} \mathrm{SL}$ is due to anharmonic phonon-phonon coupling (population decay). The damping in the amorphous phase, on the other hand, does not depend on the temperature ${ }^{27}$ and therefore would be dominated by phonon-defect scattering (pure dephasing) whose rate is proportional to the density of lattice defects (vacancy). ${ }^{18,28}$ This supports the conclusion that the randomly distributed vacancies (or voids) exist in the amorphous phase while they change into ordered structure, which would not effectively scatter coherent optical phonons. Note that the anharmonic phonon-phonon coupling would also partly contribute to the damping of the $\mathrm{A}_{1}$ mode in the amorphous phase, however, the almost flat temperature dependence of the decay rate in Fig. 4 strongly suggests that the anharmonic phonon decay path is blocked by the randomly distributed vacancies (or voids). Although the calculation of the phonon dispersion for the amorphous GeTe is not currently available, a possible reason why the anharmonic decay process is absent in the amorphous GST SLs is a breakdown in the phonon momentum conservation within the Brillouin zone. ${ }^{29,30}$

As shown in Fig. 4(a), the frequency of the coherent $A_{1}$ mode decreases as the temperature increases. This temperature dependence is qualitatively in good agreement with the anharmonic frequency shift observed by Raman-scattering measurements. ${ }^{32}$ Such a frequency shift due to the lattice anharmonicity was also observed in III-V semiconductors, which was reproduced by $a b$ initio calculations including various anharmonic contributions (thermal expansion, thirdorder, and fourth-order anharmonicity). ${ }^{33}$ The difference in the frequency between the amorphous and the crystalline phases of $\sim 0.1 \mathrm{THz}$ suggests that the local structure of $\mathrm{GeTe}_{4}$ in the amorphous phase changes into $\mathrm{GeTe}_{6}$ in the crystalline phase due to the flip-flop structural change in $\mathrm{GeTe} / \mathrm{Sb}_{2} \mathrm{Te}_{3}$ SLs. $^{6}$

To conclude, we have studied ultrafast dynamics of coherent optical phonons in $\mathrm{GeTe} / \mathrm{Sb}_{2} \mathrm{Te}_{3}$ SLs to show the damping of the coherent $\mathrm{A}_{1}$ mode is temperature dependent in crystalline phase, while that in the amorphous phase is not. These facts can be understood in terms of phonon anharmonic decay in the crystalline phase and phonon-defect (vacancy) scattering in the amorphous phase. Thus the existence of disordered vacancies (or voids) is evident in the amorphous phase while the vacancies (or voids) in the crystalline phase are highly ordered. The frequency shift of the $A_{1}$ mode observed in the amorphous phase relative to the crystalline phase is suggestive to the local structural change of $\mathrm{GeTe}_{4}$ into $\mathrm{GeTe}_{6}$. The disordering of the vacancies plays a dominant role in the volume expansion in amorphous GST film, resulting in the frequency redshift relative to the $\mathrm{GeTe} / \mathrm{Sb}_{2} \mathrm{Te}_{3}$ SLs. We believe that the present study has uncovered vivid information on the arrangement of the vacancies as well as ultrafast dephasing dynamics of lattice vibrations in $\mathrm{GeTe} / \mathrm{Sb}_{2} \mathrm{Te}_{3} \mathrm{SLs}$ toward the application of laser-induced optical switching using this unique material. This method can be applied to all the other Ge-Sb-Te systems to understand fundamental lattice dynamics.

This work was supported in part by MEXT under Grant No. KAKENHI-19540329 and "Innovation Research Project on Nanoelectronics Materials and Structures-research and development of superlatticed chalcogenide phase-change memory based on new functional structures" from METI, Japan.

\footnotetext{
*mhase@bk.tsukuba.ac.jp

${ }^{1}$ N. Yamada, E. Ohno, K. Nishiuchi, and N. Akahira, J. Appl. Phys. 69, 2849 (1991)

${ }^{2}$ N. Yamada and T. Matsunaga, J. Appl. Phys. 88, 7020 (2000).

${ }^{3}$ J. Hegedüs and S. R. Elliott, Nature Mater. 7, 399 (2008).

${ }^{4}$ Z. Sun, J. Zhou, and R. Ahuja, Phys. Rev. Lett. 96, 055507 (2006).
}

${ }^{5}$ Z. Sun, J. Zhou, and R. Ahuja, Phys. Rev. Lett. 98, 055505 (2007).

${ }^{6}$ A. V. Kolobov, P. Fons, A. I., Frenkel, A. L. Ankudinov, J. Tominaga, and T. Uruga, Nature Mater. 3, 703 (2004).

${ }^{7}$ A. V. Kolobov, J. Haines, A. Pradel, M. Ribes, P. Fons, J. Tominaga, Y. Katayama, T. Hammouda, and T. Uruga, Phys. Rev. Lett. 97, 035701 (2006). 
${ }^{8}$ D. A. Baker, M. A. Paesler, G. Lucovsky, S. C. Agarwal, and P. C. Taylor, Phys. Rev. Lett. 96, 255501 (2006).

${ }^{9}$ K. S. Andrikopoulos, S. N. Yannopoulos, A. V. Kolobov, P. Fons, and J. Tominaga, J. Phys. Chem. Solids 68, 1074 (2007).

${ }^{10}$ Z. Sun, J. Zhou, A. Blomqvist, B. Johansson, and R. Ahuja, Phys. Rev. Lett. 102, 075504 (2009).

${ }^{11}$ M. Först, T. Dekorsy, C. Trappe, M. Laurenzis, H. Kurz, and B. Béchevet, Appl. Phys. Lett. 77, 1964 (2000).

${ }^{12}$ T. C. Chong, L. P. Shi, X. Q. Wei, R. Zhao, H. K. Lee, P. Yang, and A. Y. Du, Phys. Rev. Lett. 100, 136101 (2008). The three different states of GST SLs correspond to (i) the state that both the GeTe and the $\mathrm{Sb}_{2} \mathrm{Te}_{3}$ layers are crystalline, (ii) that both the GeTe and the $\mathrm{Sb}_{2} \mathrm{Te}_{3}$ layers are amorphous, and (iii) that only the $\mathrm{Sb}_{2} \mathrm{Te}_{3}$ layer is crystallized while the GeTe layer is still amorphous.

${ }^{13}$ M. Hase, M. Kitajima, S. I. Nakashima, and K. Mizoguchi, Phys. Rev. Lett. 88, 067401 (2002).

${ }^{14}$ M. Hase, M. Kitajima, S. Nakashima, and K. Mizoguchi, Appl. Phys. Lett. 83, 4921 (2003).

${ }^{15}$ R. Lu, M. Hase, M. Kitajima, S. Nakashima, and S. Sugai, Phys. Rev. B 75, 012107 (2007).

${ }^{16}$ A. Cavalleri, Th. Dekorsy, H. H. W. Chong, J. C. Kieffer, and R. W. Schoenlein, Phys. Rev. B 70, 161102(R) (2004).

${ }^{17}$ C. Kübler, H. Ehrke, R. Huber, R. Lopez, A. Halabica, R. F. Haglund, Jr., and A. Leitenstorfer, Phys. Rev. Lett. 99, 116401 (2007).

${ }^{18}$ M. Hase, K. Ishioka, M. Kitajima, K. Ushida, and S. Hishita, Appl. Phys. Lett. 76, 1258 (2000).

${ }^{19}$ J. Tominaga, P. Fons, A. Kolobov, T. Shima, T. C. Chong, R. Zhao, H. K. Lee, and L. Shi, Jpn. J. Appl. Phys. 47, 5763
(2008); we annealed the as-grown GST films at the slightly lower temperature of $493 \mathrm{~K}\left(220^{\circ} \mathrm{C}\right)$ for $10 \mathrm{~min}$ to crystallize the films.

${ }^{20}$ J. L. F. Da Silva, A. Walsh, and H. Lee, Phys. Rev. B 78, 224111 (2008).

${ }^{21}$ P. Y. Yu and M. Cardona, Fundamentals of Semiconductors (Springer-Verlag, Berlin, 1999).

${ }^{22}$ B.-S. Lee, J. R. Abelson, S. G. Bishop, D.-H. Kang, B.-K. Cheong, and K.-B. Kim, J. Appl. Phys. 97, 093509 (2005).

${ }^{23}$ C. Guillon, J. Burgin, P. Langot, F. Vallée, and B. Hehlen, Appl. Phys. Lett. 86, 081909 (2005).

${ }^{24}$ M. Cardona, in Light Scattering in Solids, 4th ed., edited by M. Cardona and G. Güntherodt (Springer-Verlag, Berlin, 1984).

${ }^{25}$ F. Vallée, Phys. Rev. B 49, 2460 (1994).

${ }^{26}$ M. Hase, K. Mizoguchi, H. Harima, S. Nakashima, and K. Sakai, Phys. Rev. B 58, 5448 (1998).

${ }^{27}$ The decay rate in the amorphous phase decreases very slightly with increasing the temperature, implying that the arrangement of the vacancies gradually change into ordered structure toward the amorphous-crystalline phase change at $\sim 503 \mathrm{~K}$.

${ }^{28}$ K. Ishioka, M. Hase, M. Kitajima, and K. Ushida, Appl. Phys. Lett. 78, 3965 (2001).

${ }^{29}$ E. Ching-Prado, J. Cordero, R. S. Katiyar, and A. S. Bhalla, J. Vac. Sci. Technol. A 14, 762 (1996).

${ }^{30}$ M. Kitajima, Crit. Rev. Solid State Mater. Sci. 22, 275 (1997).

${ }^{31}$ R. Shaltaf, E. Durgun, J.-Y. Raty, Ph. Ghosez, and X. Gonze, Phys. Rev. B 78, 205203 (2008).

${ }^{32}$ H. Schulz and S. Hüfner, Solid State Commun. 20, 827 (1976).

${ }^{33}$ A. Debernardi, Solid State Commun. 113, 1 (1999). 